\title{
Anisotropic nanoparticles of precise microstructure polyolefins $\dagger$
}

\author{
Justyna Trzaskowski, ${ }^{a}$ Christian Rabe, ${ }^{b}$ Karsten Vogtt, ${ }^{b}$ Marina Krumova, ${ }^{a}$ \\ Guenter Goerigk, ${ }^{b}$ Matthias Ballauff ${ }^{* b}$ and Stefan Mecking ${ }^{* a}$
}

Nanoparticles of precisely branched polyethylenes possess a distinct oblate shape resulting from a crystalline lamella in the particle as revealed by SAXS and TEM, and display controllable and well-behaved thermal behaviour.

For inorganic nanoparticles it is well established that their shape and physical properties are governed by their crystalline structure and the crystallization process. ${ }^{1,2}$ Other than for inorganic materials, crystalline order in polymers results from van der Waals interactions between adjacent chain segments. Its impact on the shapes and properties of nanoparticles has been little studied.

Polyethylene is of particular interest in this regard. Its crystalline structure and crystallization in the bulk have been studied more extensively than for any other polymer, and it is of enormous practical importance. ${ }^{3}$

Insertion polymerization of ethylene in aqueous solutions afforded surfactant-stabilized dispersions of single crystal nanoparticles of $c a .6 \mathrm{~nm}$ lamella thickness and $25 \mathrm{~nm}$ lateral extension composed of linear polyethylenes. ${ }^{4}$ Likewise, crystallization of the hydrophobic core of micelles formed from block copolymers with a polyethylene and a hydrophilic block yielded platelet or disc-like particles. ${ }^{5-7}$ Self-assembly in aqueous solution of linear ethylene-acrylic acid copolymers affords crystalline particles colloidally stabilized by covalently attached carboxylate moieties. ${ }^{8}$ Anisotropic particles of moderately branched polyethylene resulted from free-radical emulsion polymerization at intermediate pressures. ${ }^{9}$

The aforementioned nanocrystals of high molecular weight linear polymers feature sharp thermal transitions $\left(\mathrm{ca} . T_{\mathrm{m}}\right.$ $130{ }^{\circ} \mathrm{C}, T_{\mathrm{c}} 75^{\circ} \mathrm{C}$ ), and recrystallize with a high supercooling due to the confined nature of the nanodroplets. A control of these transitions was not possible, however. The introduction of branches alters the melting and crystallization point, but also results in very broad thermal transitions which indicate ill-defined crystalline structures. To this end, nanoparticles composed of precisely branched polymers were investigated.

${ }^{a}$ Chair of Chemical Materials Science, Department of Chemistry, University of Konstanz, Universitätsstrasse 10, D-78457 Konstanz, Germany.E-mail: Stefan.Mecking@uni-konstanz.de;

Fax: $+49753188-5152$

${ }^{b}$ Helmholtz-Zentrum Berlin for Materials and Energy,

Hahn Meitner-Platz 1, D-14109 Berlin, Germany.

E-mail: matthias.ballauff@helmholtz-berlin.de

$\dagger$ Electronic supplementary information (ESI) available.
Bulk materials with such microstructures have recently been studied extensively by Wagener et al. ${ }^{10,11}$ Methyl-branched $\alpha, \omega$ diene monomers $\mathrm{H}_{2} \mathrm{C}=\mathrm{CH}\left(\mathrm{CH}_{2}\right)_{n} \mathrm{CHMe}\left(\mathrm{CH}_{2}\right)_{n} \mathrm{CH}=\mathrm{CH}_{2}$, $n=6: 1$ and $n=8: 2$ were prepared by a previously reported five step procedure (Fig. S1, ESI $\dagger$ ). ${ }^{10}$ GC analysis revealed a purity of $>99 \%$, which is crucial for the subsequent step-growth polymerization (Fig. S2, ESI $\dagger$ ). Acylic diene metathesis (ADMET) polymerization in a mechanically stirred microreactor with [ $\left(\mathrm{PCy}_{3}\right)_{2} \mathrm{Cl}_{2} \mathrm{Ru}=\mathrm{CHPh}$ (Grubbs' 1st generation catalyst) for six days yielded main-chain unsaturated poly-1 and poly-2, respectively. Molecular weights as determined by GPC (in THF vs. PS standards) amount to $M_{\mathrm{n}}=4.1 \times 10^{4} \mathrm{~g} \mathrm{~mol}^{-1}$, $M_{\mathrm{w}} / M_{\mathrm{n}}=1.7$ for poly-1, and $M_{\mathrm{n}}=2.9 \times 10^{4} \mathrm{~g} \mathrm{~mol}^{-1}$, $M_{\mathrm{w}} / M_{\mathrm{n}}=1.8$ for poly-2. ${ }^{1} \mathrm{H}-\mathrm{NMR}$ spectroscopic end group analysis (vinyl endgroups at $\delta 4.95$ and $5.80 \mathrm{ppm}$, and alkyl endgroups at $\delta 0.86 \mathrm{ppm}$ from isomerization during polymerization) revealed a molecular weight of $M_{\mathrm{n}} 10^{4} \mathrm{~g} \mathrm{~mol}^{-1}$ for both unsaturated polymers. This is in reasonable agreement with the apparent molecular weight from GPC of the unsaturated polymers, indicating that no excessive formation of cycles has occurred.

The double bonds in the main chain of poly-1 and poly-2 impede crystallization and enhance solubility. This is beneficial for a secondary dispersion approach to nanoparticles. The unsaturated polymers, diluted with toluene to lower the viscosity, were dispersed in an aqueous surfactant (DTAC) solution by means of ultrasonication. Dynamic light scattering revealed an average particle size of $c a .35 \mathrm{~nm}$ with a particle size distribution in the range from $0.12-0.26$ (Fig. 1).

For hydrogenation, the ruthenium alkylidene catalyst was quenched with ethyl vinyl ether to the Fischer carbene, in order to suppress any undesired secondary metathesis activity, which could enhance for example formation of macrocycles and alteration of molecular weights. Hydrogenation of the dispersion at 60 bar $\mathrm{H}_{2}$ pressure at $65{ }^{\circ} \mathrm{C}$ proceeded virtually completely (Scheme 1), as concluded from the absence of olefin
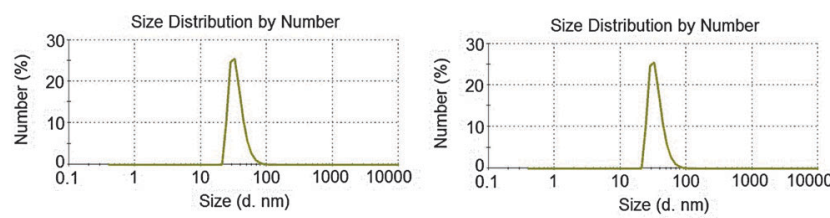

Fig. 1 Particle size distribution by number of poly-2 secondary dispersion before (left) and after (right) hydrogenation. 


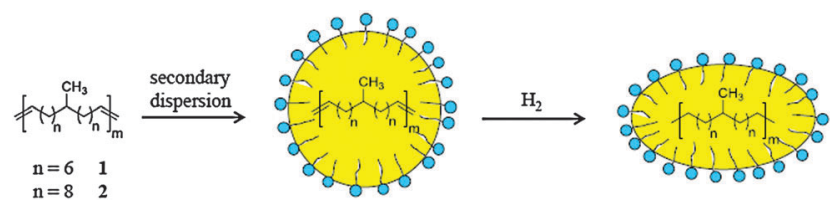

Scheme 1 Preparation of nanoparticles of precisely methyl-branched polyethylene.

resonances at $\delta 5.35 \mathrm{ppm}$ in ${ }^{1} \mathrm{H}-\mathrm{NMR}$ spectra of isolated bulk material $\mathbf{C 1 5}$ and C19, respectively (Fig. S6 and S8, ESI $\dagger$ ). This corresponds to a degree of hydrogenation $>99.9 \%$, which fulfills a prerequisite for meaningful studies of physical properties (vide infra). Particle sizes remain unaltered within experimental error during the hydrogenation procedure, indicating that the reaction occurs in individual particles which preserve their identity (Fig. 1). Also, apparent molecular weights from GPC are not altered significantly $\left(\mathbf{C 1 5}: M_{\mathrm{n}}=3.2 \times 10^{4} \mathrm{~g} \mathrm{~mol}^{-1}\right.$, $M_{\mathrm{w}} / M_{\mathrm{n}}=1.5$ and $\mathbf{C 1 9}: M_{\mathrm{n}}=3.0 \times 10^{4} \mathrm{~g} \mathrm{~mol}^{-1}, M_{\mathrm{w}} / M_{\mathrm{n}}=$ 1.7) and correspond to absolute molecular weights determined by alkyl endgroup analysis (resonance at $\delta 0.86 \mathrm{ppm}$ ) in ${ }^{1} \mathrm{H}$-NMR spectra $\left(M_{\mathrm{n}} 10^{4} \mathrm{~g} \mathrm{~mol}^{-1}\right)$. The nanoparticles, C15-NP and C19-NP, were investigated by differential scanning calorimetry (DSC) in dispersion and for comparison in bulk, which reveals their crystalline nature (Fig. 2).

Melting in the particles and recrystallization occur without any advert effect on colloidal stability. After five melting and recrystallization cycles, the particle size was unaltered (Fig. S10, ESI $†$ ). Moreover, narrow melt and crystallization transitions occur. In this respect the nanoparticles resemble linear polyethylene
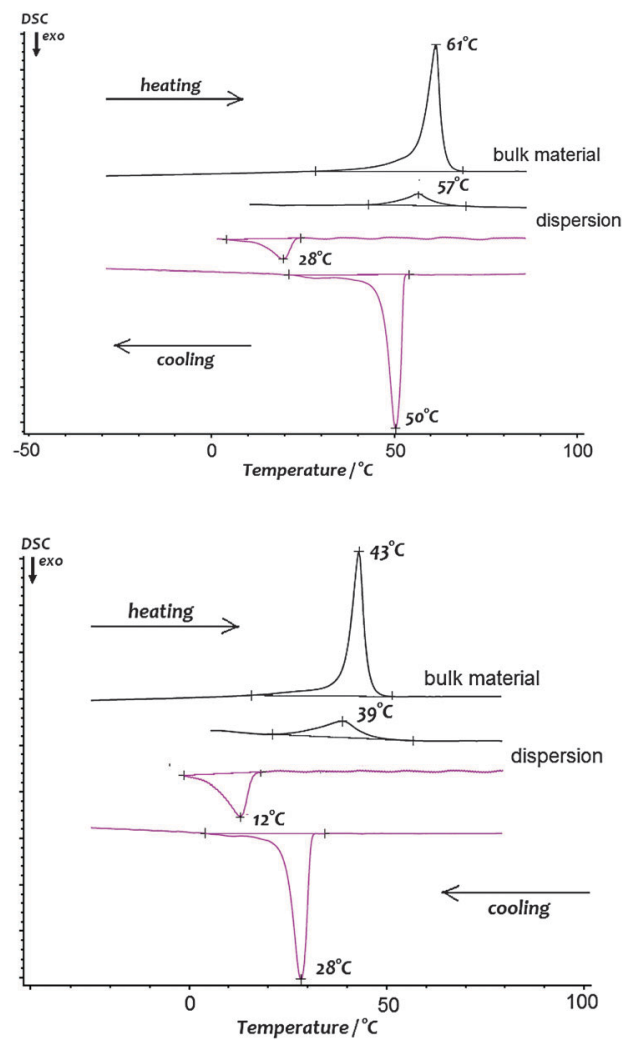

Fig. 2 DSC traces of nanoparticle dispersions C19-NP (top) and C15-NP (bottom), and bulk material for comparison $\left(10 \mathrm{~K} \mathrm{~min}^{-1}\right.$, 2nd heating cycle). nanocrystals, however the crystalline nanoparticles' thermal transitions can be tuned over a wide range down to nearly $100{ }^{\circ} \mathrm{C}$ below the melting point of linear polyethylene, with $T_{\mathrm{m}}=57{ }^{\circ} \mathrm{C}$ for C19-NP and $T_{\mathrm{m}}=39{ }^{\circ} \mathrm{C}$ for C15-NP. By comparison, nanoparticles of a polyethylene similar to $\mathrm{C19}$ in its branching structure but with random placement of branches are essentially non-crystalline (which also reflects in a spherical shape, cf. below) ${ }^{12}$ Crystallization of nanoparticles occurs with a high supercooling of $c a .28{ }^{\circ} \mathrm{C}$, due to crystallization of individual droplets, vs. typical heterogeneous nucleation in the bulk.

Small angle X-ray scattering (SAXS) in combination with contrast variation can reveal the dimensions of polymer particles in this small size regime. ${ }^{4,13,14}$ The small angle scattering intensities of C19-NP were studied in neat water and in glucose solutions in a contrast variation series (Fig. S12, ESI $\dagger$ ). ${ }^{15}$ Due to a non-negligible scattering contribution of DTAC-micelles the analysis was only suitable for $q$-values lower than $0.7 \mathrm{~nm}^{-1}$ (refer to ESI $\dagger$ ).

From a Guinier analysis of the scattering intensities at low $q$-values (Fig. S13, ESI $\dagger$ ) the initial parameters of the polyethylene nanoparticles were extracted (see ESI $\dagger$; Table S2). ${ }^{15,16}$ Further analysis of the data yielded the average particle volume $V_{\mathrm{P}}=36000 \pm 3000 \mathrm{~nm}^{3}$ and the average particle scattering length density $\rho_{\mathrm{P}}=8.71 \pm 0.0410^{10} \mathrm{~cm}^{-2}$, respectively.

For a more detailed determination of the shape of the particles and their internal structure, the scattering intensities $I(q)$ were decomposed as a function of the contrast $\Delta \rho .^{15,17-20}$ This technique allows for the determination of the particles' outer shape by its specific scattering contribution unaffected by the contribution arising from the particles inner structure ( $c f$. ESI $\dagger)$.

Fig. 3 displays the scattering intensities normalized by the volume fraction $\phi$ and the contrast $\Delta \rho$. This analysis shows that particles are not homogeneous but exhibit a variation of electron density in their interior. This can be argued directly from the shallow maximum at around $q \sim 0.4 \mathrm{~nm}^{-1}$ (Fig. 3). We ascribe this finding to a crystalline core of the particles, which is further corroborated by TEM (vide infra). Unfortunately, the polydispersity of the particles is too high to analyse the internal variation of electron density by SAXS in further detail. However, the data were of sufficient quality to extrapolate the scattering intensities in infinite contrast. The resulting partial intensity $I_{\mathrm{S}}(q)$

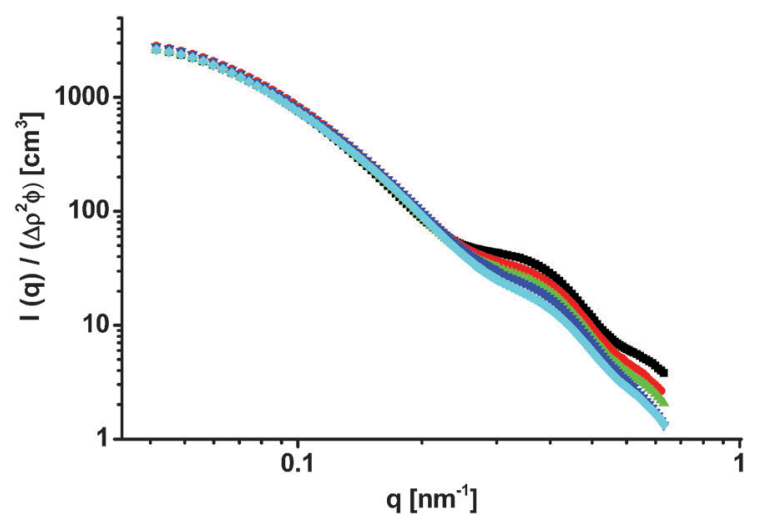

Fig. 3 Scattering intensities $I(q)$ from a contrast variation series of C19-NP normalized by volume fraction $\phi$ and contrast $\Delta \rho$. The data are background corrected for contributions of glucose and DTAC (see $\mathrm{ESI} \dagger$ for further details). 


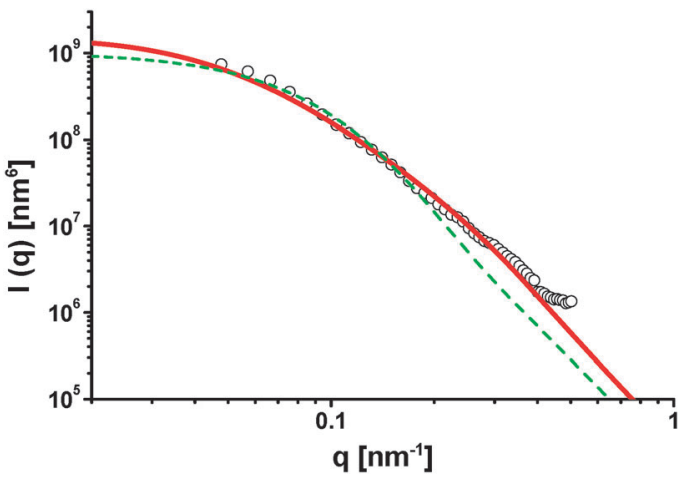

Fig. 4 Scattering contribution of the shape-term $I_{\mathrm{S}}(q)$ derived from the contrast decomposition (open symbols). The corresponding scattering intensities of an oblate ellipsoid were obtained by modelling (red line). For comparison, a fit for polydisperse spheres is shown (dashed line).

refers solely to the shape of the particles. Fig. 4 displays $I_{\mathrm{S}}(q)$ together with the corresponding fit. $I_{\mathrm{S}}(q)$ could be modelled best by a polydisperse oblate-like spheroid with an equatorial-axis with $r_{\text {eq }}=23.8 \pm 8.8 \mathrm{~nm}$ and a perpendicular semi-axis with $r_{\mathrm{p}}=$ $4.9 \pm 1.8 \mathrm{~nm}$ (Fig. 4). This fit also affords the correct particle volume in agreement with the Guinier-fit (vide supra). For comparison, the best fit for polydisperse spheres (with $d=$ $27 \pm 10 \mathrm{~nm}$ ) shows significant deviations from the decomposed shape function in the high- $q$ region and can be ruled out.

Further insight into the particle internal structure was obtained from transmission electron microscopy (TEM) (Fig. 5). The nanoparticles appear to consist of one $c a$. $10 \mathrm{~nm}$ thick lamella which is mainly on the edge, that is, perpendicular to the longer particle axis, embedded in an amorphous matrix. This renders the particles a distinctly anisotropic shape, as also confirmed by tilting of the sample in the electron microscope (Fig. 5). The ellipsoids observed by TEM possess an equatorial diameter of $c a .50 \mathrm{~nm}$ and a height of $c a$. 10 to $20 \mathrm{~nm}$, which agrees well with the SAXS data. This particle structure, which differs from the bulk morphology, ${ }^{21}$ is not easily understood given that the polymer is not only homogeneous regarding its molecular structure (precisely placed branches) but also molecular weight distributions are well behaved (there are no fractions with extremely different molecular weights that might differ in the crystallization behaviour). Our current
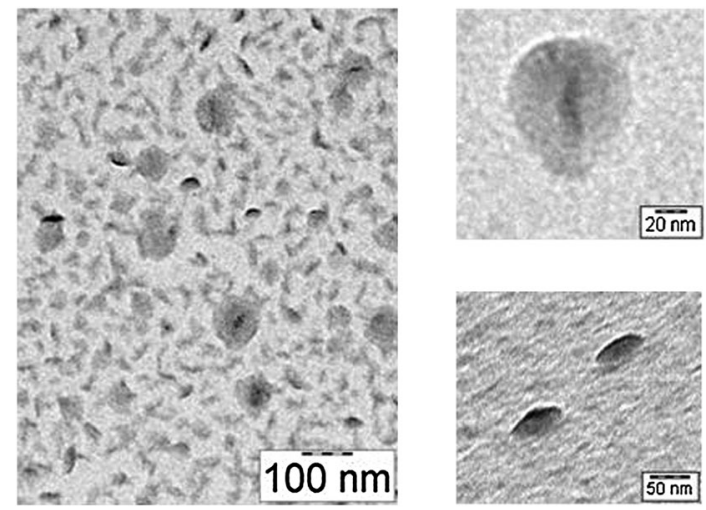

Fig. 5 TEM images of nanoparticle dispersion C19-NP. Left: overview; right: close-up of particle (top), sample tilted by $60^{\circ}$ (bottom). understanding is that this might arise from the crystallization process in the nanoscale droplets. A lamella forms by crystallization with its thickness dimension defined by the crystallization conditions, essentially by the temperature. Due to confinement in the droplet and particle, respectively, material is not incorporated into the lamella but is left as surrounding amorphous phase.

In conclusion, the crystallization of precisely branched linear polyethylene in nanoscale droplets provides particles with a distinct oblate shape. This is found independently by $\mathrm{X}$-ray scattering and electron microscopy. The strongly anisotropic nature appears to arise from a crystalline lamella in the particles, which also imparts shape upon the amorphous phase, present in an approximately equal volume as the crystalline portion. The strongly anisotropic nature appears to arise from a crystalline lamella in the particles, which also imparts shape upon the amorphous phase, present in an approximately equal volume as the crystalline portion.

The authors would like to thank Dr Narayanan from the ID02 beamline at the ESRF in Grenoble (France) for the kind help with the SAXS measurements. J. T. gratefully acknowledges a stipend provided by the DFG (IGK 667).

\section{Notes and references}

1 S. L. Shen and X. Wang, Chem. Commun., 2010, 46, 6891-6899.

2 Y. G. Sun and Y. N. Xia, Science, 2002, 298, 2176-2179.

3 G.-R. Strobl, Physics of polymers concepts for understanding their structures and behavior, Springer, Berlin, 3rd edn, 1997.

4 C. H. M. Weber, A. Chiche, G. Krausch, S. Rosenfeldt, M. Ballauff, L. Harnau, I. Goettker-Schnetmann, Q. Tong and S. Mecking, Nano Lett., 2007, 7, 2024-2029.

5 L. G. Yin and M. A. Hillmyer, Macromolecules, 2011, 44, 3021-3028.

6 T. Li, W. J. Wang, R. Liu, W. H. Liang, G. F. Zhao, Z. Y. Li, Q. Wu and F. M. Zhu, Macromolecules, 2009, 42, 3804-3810.

7 H. Schmalz, J. Schmelz, M. Drechsler, J. Yuan, A. Walther, K. Schweimer and A. M. Mihut, Macromolecules, 2008, 41, 3235-3242.

8 V. A. Kryuchkov, J. C. Daigle, K. M. Skupov, J. P. Claverie and F. M. Winnik, J. Am. Chem. Soc., 2010, 132, 15573-15579.

9 E. Grau, P. Y. Dugas, J. P. Broyer, C. Boisson, R. Spitz and V. Monteil, Angew. Chem., Int. Ed., 2010, 49, 6810-6812.

10 J. A. Smith, K. R. Brzezinska, D. J. Valenti and K. B. Wagener, Macromolecules, 2000, 33, 3781-3794.

11 M. E. Seitz, C. D. Chan, K. L. Opper, T. W. Baughman, K. B. Wagener and K. I. Winey, J. Am. Chem. Soc., 2010, 132, 8165-8174.

12 Q. Tong, M. Krumova, I. Goettker-Schnetmann and S. Mecking, Langmuir, 2008, 24, 2341-2347.

13 C. N. Rochette, S. Rosenfeldt, K. Henzler, F. Polzer, M. Ballauff, Q. Tong, S. Mecking, M. Drechsler, T. Narayanan and L. Harnau, Macromolecules, 2011, 44, 4845-4851.

14 J. Bolze, M. Ballauff, T. Rische, D. Rudhardt and A. Meixner, Macromol. Chem. Phys., 2004, 205, 165-172.

15 L. A. S. Feigin and D. I. Svergun, Structure analysis by small-angle $X$-ray and neutron scattering, Plenum Press, New York, 1987.

16 H. B. Stuhrman and R. G. Z. Kirste, J. Phys. Chem., 1967, 56, 334-337.

17 M. Ballauff, Adv. Eng. Mater., 2011, 13, 793-802.

18 S. Rosenfeldt, E. Karpuk, M. Lehmann, H. Meier, P. Lindner, L. Harnau and M. Ballauff, ChemPhysChem, 2006, 7, 2097-2104.

19 N. Dingenouts, J. Bolze, D. Potschke and M. Ballauff, Adv. Polym. Sci., 1999, 144, 1-47.

20 P. Hickl, M. Ballauff and A. Jada, Macromolecules, 1996, 29, 4006-4014.

21 G. Lieser, G. Wegner, J. A. Smith and K. B. Wagener, Colloid Polym. Sci., 2004, 282, 773-781. 